\title{
Calorie labeling and consumer estimation of calories purchased
}

Glen B Taksler ${ }^{1 *}$ and Brian Elbel ${ }^{2,3}$

\begin{abstract}
Background: Studies rarely find fewer calories purchased following calorie labeling implementation. However, few studies consider whether estimates of the number of calories purchased improved following calorie labeling legislation.

Findings: Researchers surveyed customers and collected purchase receipts at fast food restaurants in the United States cities of Philadelphia (which implemented calorie labeling policies) and Baltimore (a matched comparison city) in December 2009 (pre-implementation) and June 2010 (post-implementation). A difference-in-difference design was used to examine the difference between estimated and actual calories purchased, and the odds of underestimating calories.

Participants in both cities, both pre- and post-calorie labeling, tended to underestimate calories purchased, by an average 216-409 calories. Adjusted difference-in-differences in estimated-actual calories were significant for individuals who ordered small meals and those with some college education (accuracy in Philadelphia improved by 78 and 231 calories, respectively, relative to Baltimore, $p=0.03-0.04)$. However, categorical accuracy was similar; the adjusted odds ratio [AOR] for underestimation by $>100$ calories was $0.90(p=0.48)$ in difference-in-difference models. Accuracy was most improved for subjects with a BA or higher education ( $A O R=0.25, p<0.001$ ) and for individuals ordering small meals ( $A O R=0.54, p=0.001)$. Accuracy worsened for females $(A O R=1.38, p<0.001)$ and for individuals ordering large meals $(A O R=1.27, p=0.028)$.
\end{abstract}

Conclusions: We concluded that the odds of underestimating calories varied by subgroup, suggesting that at some level, consumers may incorporate labeling information.

Keywords: Diet, Health policy, Energy intake, Caloric restriction, Obesity

Calorie labeling legislation has been introduced in several United States cities and states to reduce obesity rates. Nationally, the Patient Protection and Affordable Care Act is expected to require restaurants with $\geq 20$ locations to post calories for all regular food and drink items [1].

Yet, studies suggest that calorie labeling has little impact on the number of calories purchased. Studies from Philadelphia [2] and low-income areas in New York City [3] found that labeling was associated with consumers noticing calorie labels but no significant change in calories purchased. Most other controlled studies have found similar results [4-7], although one study found that consumers at Starbucks purchased 12 fewer calories

\footnotetext{
*Correspondence: taksleg@ccforg

'Medicine Institute, Cleveland Clinic, 9500 Euclid Avenue, G1-40F, Cleveland, $\mathrm{OH}$ 44195, USA

Full list of author information is available at the end of the article
}

following calorie labeling [8]. Experimental studies have found mixed results $[9,10]$.

Despite little evidence of a change in number of calories purchased, recent work has considered whether labeling is associated with greater accuracy in estimates of the number of calories purchased [11]. That is, while consumers purchase a similar number of calories, do they better judge the caloric content of foods following labeling policies? Such a finding could indicate that, at some level, consumers absorb calorie labeling information. Given the time associated with behavior change, such a mechanism could indicate an important first step in the potential longerterm impact of labeling. One prior study suggests that consumers were 9 percentage points more accurate in correctly predicting calories purchased (within 100 calories, from $15 \%$ before labeling to $24 \%$ after labeling) [11], but was limited to New York City. Other prior work 
has attributed caloric underestimation to a lack of visual cues $[12,13]$. In one study, subjects who ate from self-refilling soup bowls (lacking the visual control of a bowl for portion size) were found to consume $73 \%$ more soup than controls; however, both groups estimated similar caloric consumption [12]. Caloric underestimation may also be related to nutritional status (overestimation of energy content for unhealthy foods) [14], less overall health consciousness [15], and lower education [16]. More generally, food labels appear most often used when easier-to-understand $[17,18]$, though some literature suggests an association to health literacy [19-22], female gender [21-23], and higher education [21,22].

Using a larger and more diverse sample than previous research, researchers examine the influence of calorie labeling on estimation of calories purchased in Philadelphia.

\section{Findings \\ Methods}

Data were collected as part of a larger study to examine the influence of calorie labeling implemented in Philadelphia in 2010 [2]. A difference-in-difference design was used to examine the difference between estimated and actual calories purchased in Philadelphia in December 2009 (pre-calorie labeling) versus June 2010 (post-calorie labeling), as compared to Baltimore (a matched comparison city without calorie labeling rules) during the same month. The Appendix describes difference-in-difference methodology in more detail. Baltimore was selected as the city most comparable to Philadelphia by calculating Euclidean distances between Philadelphia and each of the largest 100 US cities using standardized city-level measures derived from Census 2000 data, including population size, poverty, unemployment, education, race/ethnicity, and income measures [2]. Full methods are available elsewhere [2].

Research staff stood outside locations of McDonald's and Burger King during lunch (approximately 11:30 am2:30 pm) or dinner (approximately 5:00 pm-8:00 pm) on weekdays, and approached entering customers appearing to be $\geq 18$ years old and asked them to bring back their receipt in exchange for \$2 [2]. Participants who agreed were asked questions including which items were ordered for him/herself (versus other individuals); the exact nature of items (added cheese, mayonnaise, etc.); how often they visited "big chain" fast food restaurants; and how many calories they estimated to be in their purchase. The receipt provided was used to calculate actual calories purchased, based on nutrition information provided by each restaurant (as of May 2010) [2].

First, summary statistics were calculated for the full sample $(\mathrm{N}=1835)$ and subgroups based on number of calories purchased ( $\leq$ median [850 calories] vs. >median), gender, race/ethnicity, education, and food vs. beverage.
Summary statistics were calculated for each city, both pre- and post-calorie labeling. T-tests of unadjusted statistical significance were run for 4 groups: Philadelphia vs. Baltimore pre-calorie labeling, Philadelphia vs. Baltimore post-calorie labeling, Philadelphia pre- versus post-calorie labeling, and Baltimore pre- versus post-calorie labeling.

Researchers then examined the difference between estimated and actual calories using multiple regression models. The dependent variable was estimated minus actual calories for each respondent. A positive number meant an overestimate and a negative number meant an underestimate of actual calories. The key independent variable of interest was an interaction term between Philadelphia (versus Baltimore) and post-calorie labeling (versus pre-calorie labeling). That is, researchers sought to measure the marginal contribution of calorie labeling policies to the accuracy of estimates in Philadelphia. Independent covariates included age, gender, race/ethnicity, education, number of items purchased, purchase of a combination meal, to-go vs. eat-in consumption, number of fast food restaurant visits per week, city, and time period (pre- vs. post-calorie labeling).

Finally, consistent with prior research suggesting that consumers tend to underestimate calories $[2,3,11,24]$, logistic regression models were used to consider whether subjects underestimated by $>100,>250$, and $>500$ calories. (Researchers verified that consumers in the sample, on average, underestimated calories; results shown below.) This analysis was used to consider broad patterns in accuracy pre- vs. post-calorie labeling, as opposed to the magnitude difference between estimated and actual calories. Odds ratios were adjusted for the same covariates described above.

Standard errors were clustered by restaurant. Tests were performed with a two-sided alpha $=0.05$. This study was approved by the Institutional Review Board of New York University School of Medicine.

\section{Results}

Table 1 presents summary statistics. Respondents were primarily male, black or African American, and held a high school or lower education. No significant differences were observed in the actual number of calories purchased, though some differences existed across cities (a larger proportion of females in Philadelphia, and larger proportion of blacks and fast food visits/week in Baltimore) and time periods (a larger proportion of females and blacks in Philadelphia, and less missing data in Baltimore, in the post-calorie labeling period).

Table 2 shows regression results for the difference between estimated and actual calories. In the full sample and every subgroup, participants in both cities and time periods tended to underestimate calories purchased, by an average of 216-409 calories. The difference-in-difference coefficient 
Table 1 Summary statistics

\begin{tabular}{|c|c|c|c|c|c|c|c|c|c|c|c|c|c|c|}
\hline & \multirow{3}{*}{$\begin{array}{l}\text { All } \\
\text { Mean }\end{array}$} & \multirow[b]{3}{*}{ SD } & \multicolumn{4}{|c|}{ Philadelphia } & \multicolumn{4}{|c|}{ Baltimore } & \multicolumn{4}{|c|}{ Significance tests } \\
\hline & & & \multicolumn{2}{|l|}{ Pre- } & \multicolumn{2}{|l|}{ Post- } & \multicolumn{2}{|l|}{ Pre- } & \multicolumn{2}{|l|}{ Post- } & \multirow[t]{2}{*}{ Pre- } & \multirow[t]{2}{*}{ Post- } & \multicolumn{2}{|l|}{ Pre vs. Post } \\
\hline & & & Mean & SD & Mean & SD & Mean & SD & Mean & SD & & & Philadelphia & Baltimore \\
\hline N & 1835 & & 470 & & 534 & & 394 & & 437 & & & & & \\
\hline \multicolumn{15}{|l|}{ Mean } \\
\hline Age & 39.1 & 13.9 & 39.7 & 14.1 & 37.4 & 14.4 & 40.8 & 13.5 & 38.9 & 13.4 & & & * & * \\
\hline Number of calories purchased, actual & 951 & 685 & 987 & 757 & 927 & 704 & 974 & 696 & 923 & 559 & & & & \\
\hline \multicolumn{15}{|l|}{ Percent } \\
\hline \multicolumn{15}{|l|}{ Gender } \\
\hline Male & 55.2 & 49.8 & 58.3 & 49.4 & 52.1 & 50.0 & 51.5 & 50.0 & 58.8 & 49.3 & * & * & * & \\
\hline Female & 37.4 & 48.4 & 37.5 & 48.5 & 45.5 & 49.8 & 28.9 & 45.4 & 35.2 & 47.8 & $* *$ & $* *$ & $* *$ & \\
\hline Missing & 7.4 & 26.2 & 4.3 & 20.2 & 2.4 & 15.4 & 19.5 & 39.7 & 6.0 & 23.7 & $* * *$ & $* *$ & & $* * *$ \\
\hline \multicolumn{15}{|l|}{ Race/Ethnicity } \\
\hline Black & 70.1 & 45.8 & 60.4 & 49.0 & 70.8 & 45.5 & 73.9 & 44.0 & 76.4 & 42.5 & $* * *$ & * & $* * *$ & \\
\hline Caucasian & 20.8 & 40.6 & 23.0 & 42.1 & 17.8 & 38.3 & 22.1 & 41.5 & 21.1 & 40.8 & & & * & \\
\hline Other/Missing & 4.1 & 19.8 & 6.0 & 23.7 & 5.2 & 22.3 & 3.3 & 17.9 & 1.4 & 11.7 & & $* *$ & & \\
\hline \multicolumn{15}{|l|}{ Education } \\
\hline High school or less & 60.9 & 48.8 & 54.7 & 49.8 & 63.3 & 48.2 & 62.2 & 48.6 & 63.4 & 48.2 & * & & ** & \\
\hline Some college or AA & 25.2 & 43.4 & 29.8 & 45.8 & 23.0 & 42.1 & 22.6 & 41.9 & 25.2 & 43.5 & * & & * & \\
\hline BA or above & 10.6 & 30.8 & 11.7 & 32.2 & 8.1 & 27.2 & 12.4 & 33.0 & 10.8 & 31.0 & & & & \\
\hline Missing & 3.4 & 18.1 & 3.8 & 19.2 & 5.6 & 23.1 & 2.8 & 16.5 & 0.7 & 8.3 & & $* * *$ & & * \\
\hline \multicolumn{15}{|l|}{ Type of order } \\
\hline To go & 67.6 & 46.8 & 60.6 & 48.9 & 70.4 & 45.7 & 68.0 & 46.7 & 71.4 & 45.2 & * & & $* *$ & \\
\hline Eat in & 26.3 & 44.1 & 25.1 & 43.4 & 24.2 & 42.8 & 28.4 & 45.2 & 28.4 & 45.1 & & & & \\
\hline Missing & 6.1 & 23.9 & 14.3 & 35.0 & 5.4 & 22.7 & 3.6 & 18.5 & 0.2 & 4.8 & $* * *$ & $* * *$ & $* * *$ & $* * *$ \\
\hline \multicolumn{15}{|c|}{ Number of times usually eat in big chain fast food restaurant per week } \\
\hline$\leq 1$ & 56.4 & 49.6 & 62.3 & 48.5 & 64.0 & 48.0 & 49.0 & 50.1 & 47.1 & 50.0 & $* * *$ & $* * *$ & & \\
\hline 2 & 15.8 & 36.4 & 12.3 & 32.9 & 12.7 & 33.4 & 17.5 & 38.1 & 21.5 & 41.1 & * & $* * *$ & & \\
\hline$\geq 3$ & 34.4 & 47.5 & 26.4 & 44.1 & 30.7 & 46.2 & 39.6 & 49.0 & 42.8 & 49.5 & $* * *$ & $* * *$ & & \\
\hline Missing & 3.1 & 17.4 & 7.7 & 26.6 & 2.4 & 15.4 & 1.5 & 12.3 & 0.5 & 6.8 & $* * *$ & * & $* * *$ & \\
\hline \multicolumn{15}{|l|}{ Number of items purchased } \\
\hline 1 & 23.2 & 42.2 & 18.5 & 38.9 & 25.7 & 43.7 & 25.9 & 43.9 & 22.7 & 41.9 & ** & & $* *$ & \\
\hline 2 & 20.0 & 40.0 & 21.5 & 41.1 & 18.9 & 39.2 & 19.0 & 39.3 & 20.4 & 40.3 & & & & \\
\hline 3 & 31.6 & 46.5 & 31.7 & 46.6 & 32.2 & 46.8 & 31.0 & 46.3 & 31.4 & 46.4 & & & & \\
\hline 4 & 11.3 & 31.7 & 10.2 & 30.3 & 9.2 & 28.9 & 12.2 & 32.8 & 14.4 & 35.2 & & * & & \\
\hline$\geq 5$ & 14.0 & 34.7 & 18.1 & 38.5 & 14.0 & 34.8 & 11.9 & 32.5 & 11.2 & 31.6 & * & & & \\
\hline Purchased combination meal & 24.5 & 43.0 & 21.5 & 41.1 & 25.7 & 43.7 & 25.4 & 43.6 & 25.6 & 43.7 & & & & \\
\hline \multicolumn{15}{|l|}{ Restaurant } \\
\hline McDonald's & 64.2 & 48.0 & 66.2 & 47.4 & 70.2 & 45.8 & 61.7 & 48.7 & 57.0 & 49.6 & & $* * *$ & & \\
\hline Burger King & 35.8 & 48.0 & 33.8 & 47.4 & 29.8 & 45.8 & 38.3 & 48.7 & 43.0 & 49.6 & & $* * *$ & & \\
\hline
\end{tabular}

***P $<0.001,{ }^{* * P}<0.01, * P<0.05$.

was typically positive, meaning that respondents in Philadelphia were more accurate relative to Baltimore post-calorie labeling, but was only significant for 2 subgroups: respondents who purchased $\leq$ median number of calories (coefficient $=78, \mathrm{p}=0.04$ ) and respondents with some college education (coefficient $=231, \mathrm{p}=0.03$ ).

Table 3 shows the logistic regression results for subjects' likelihood to underestimate calories, versus overestimating 
Table 2 Actual versus estimated calories, Philadelphia versus Baltimore

\begin{tabular}{|c|c|c|c|c|c|c|c|c|c|c|c|}
\hline & \multicolumn{2}{|c|}{ Actual } & \multicolumn{2}{|c|}{ Estimated } & \multicolumn{4}{|c|}{ Estimated minus actual } & \multicolumn{3}{|c|}{ Difference-in-Difference } \\
\hline & \multirow[t]{2}{*}{ Pre } & \multirow[t]{2}{*}{ Post } & \multirow[t]{2}{*}{ Pre } & \multirow[t]{2}{*}{ Post } & \multirow[t]{2}{*}{ Pre } & \multirow[t]{2}{*}{ Post } & \multicolumn{2}{|c|}{ Significance tests } & \multirow[t]{2}{*}{ Unadj } & \multirow[t]{2}{*}{ Adj $(95 \% \mathrm{Cl})$} & \multirow[t]{2}{*}{$\mathbf{P}$} \\
\hline & & & & & & & Pre & Post & & & \\
\hline \multicolumn{12}{|l|}{ Full sample } \\
\hline Philadelphia & 987 & 927 & 578 & 581 & -409 & -346 & ** & & 177 & $122(-809,1052)$ & 0.35 \\
\hline Baltimore & 974 & 923 & 758 & 593 & -216 & -330 & & & & & \\
\hline \multicolumn{12}{|c|}{ Purchased $>850$ calories (median) } \\
\hline Philadelphia & 1480 & 1450 & 780 & 758 & -700 & -692 & * & & 223 & $191(-2301,2682)$ & 0.51 \\
\hline Baltimore & 1430 & 1390 & 1032 & 777 & -398 & -613 & & & & & \\
\hline \multicolumn{12}{|c|}{ Purchased $\leq 850$ calories (median) } \\
\hline Philadelphia & 446 & 459 & 357 & 422 & -89 & -37 & & & 105 & $78(20,136)$ & 0.04 \\
\hline Baltimore & 463 & 486 & 450 & 420 & -13 & -65 & & & & & \\
\hline \multicolumn{12}{|l|}{ Male $^{1}$} \\
\hline Philadelphia & 982 & 943 & 575 & 609 & -407 & -334 & & & 130 & $124(-998,1245)$ & 0.39 \\
\hline Baltimore & 1006 & 968 & 692 & 597 & -314 & -370 & & & & & \\
\hline \multicolumn{12}{|l|}{ Female $^{1}$} \\
\hline Philadelphia & 987 & 925 & 602 & 562 & -385 & -363 & & & -41 & $-87(-386,213)$ & 0.17 \\
\hline Baltimore & 993 & 834 & 689 & 591 & -305 & -243 & & & & & \\
\hline \multicolumn{12}{|l|}{ Black $^{1}$} \\
\hline Philadelphia & 933 & 858 & 543 & 585 & -389 & -273 & & & 173 & $100(-760,959)$ & 0.38 \\
\hline Baltimore & 1007 & 895 & 745 & 577 & -262 & -318 & & & & & \\
\hline \multicolumn{12}{|l|}{ White $^{1}$} \\
\hline Philadelphia & 1088 & 990 & 684 & 751 & -405 & -239 & & & 384 & $250(-524,1025)$ & 0.15 \\
\hline Baltimore & 886 & 950 & 815 & 661 & -71 & -290 & & & & & \\
\hline High school or & & & & & & & & & & & \\
\hline Philadelphia & 968 & 885 & 545 & 475 & -423 & -409 & & & 169 & $54(-590,698)$ & 0.48 \\
\hline Baltimore & 954 & 934 & 698 & 523 & -256 & -411 & & & & & \\
\hline Some college $\mathrm{c}$ & & & & & & & & & & & \\
\hline Philadelphia & 1028 & 977 & 582 & 811 & -447 & -166 & & & 170 & $231(77,385)$ & 0.03 \\
\hline Baltimore & 1065 & 914 & 758 & 718 & -307 & -196 & * & & & & \\
\hline BA or above ${ }^{1}$ & & & & & & & & & & & \\
\hline Philadelphia & 1065 & 1141 & 650 & 696 & -414 & -445 & * & & 149 & $231(-2138,2600)$ & 0.43 \\
\hline Baltimore & 968 & 900 & 919 & 671 & -49 & -229 & & & & & \\
\hline Food only & & & & & & & & & & & \\
\hline Philadelphia & 801 & 691 & 521 & 528 & -279 & -163 & & & 180 & $205(-514,924)$ & 0.17 \\
\hline Baltimore & 774 & 719 & 618 & 500 & -156 & -219 & & & & & \\
\hline Beverage only & & & & & & & & & & & \\
\hline Philadelphia & 203 & 308 & 204 & 231 & 1 & -77 & & & -13 & $-60(-1450,1329)$ & 0.68 \\
\hline Baltimore & 306 & 368 & 341 & 338 & 35 & -31 & & & & & \\
\hline Purchased 1 ite & & & & & & & & & & & \\
\hline Philadelphia & 320 & 316 & 221 & 286 & -99 & -30 & $* *$ & & 167 & $181(-864,1226)$ & 0.27 \\
\hline Baltimore & 319 & 339 & 364 & 286 & 45 & -53 & & & & & \\
\hline Purchased $>1 \mathrm{i}$ & & & & & & & & & & & \\
\hline Philadelphia & 1139 & 1138 & 660 & 683 & -480 & -455 & * & & 128 & $112(-932,1156)$ & 0.40 \\
\hline Baltimore & 1202 & 1093 & 895 & 683 & -307 & -411 & & & & & \\
\hline
\end{tabular}


Table 2 Actual versus estimated calories, Philadelphia versus Baltimore (Continued)

\begin{tabular}{|c|c|c|c|c|c|c|c|c|c|c|}
\hline \multicolumn{11}{|c|}{ Purchased combination meal } \\
\hline Philadelphia & 1441 & 1512 & 768 & 738 & -674 & -774 & & 9 & $-15(-2050,2019)$ & 0.94 \\
\hline Baltimore & 1482 & 1383 & 932 & 723 & -550 & -659 & & & & \\
\hline \multicolumn{11}{|c|}{ Did not purchase combination meal } \\
\hline Philadelphia & 863 & 725 & 527 & 527 & -337 & -198 & ** & 252 & $167(-539,872)$ & 0.20 \\
\hline Baltimore & 801 & 764 & 698 & 548 & -102 & -216 & * & & & \\
\hline
\end{tabular}

${ }^{1}$ May not sum to the full sample because of missing gender, race, and/or education for some subjects.

Unadj: Unadjusted. Adj: Adjusted.

${ }^{* *} \mathrm{P}<0.01$, ${ }^{*} \mathrm{P}<0.05$.

or correctly estimating calories. In the full sample, the odds of underestimation by $>100$ calories was similar post- vs. pre-calorie labeling legislation, with an adjusted odds ratio[AOR] of $0.90(95 \%=0.67-1.21, \mathrm{p}=0.48)$. However, gross underestimates were less likely; the AOR for underestimation by $>500$ calories was $0.75(95 \% \mathrm{CI}=0.73-0.77$, $\mathrm{p}<0.001)$. Accuracy in Philadelphia post-calorie labeling was most improved for subjects with a BA or higher education $(\mathrm{AOR}=0.25,95 \% \mathrm{CI}=0.12-0.50, \mathrm{p}<0.001)$ and for subjects ordering less than the median number of calories $(\mathrm{AOR}=0.54,95 \% \mathrm{CI}=0.37-0.78, \mathrm{p}=0.001)$. Accuracy deteriorated among females $(\mathrm{AOR}=1.38, \mathrm{p}<0.001)$, respondents who purchased more than the median number of calories $(\mathrm{AOR}=1.27, \mathrm{p}=0.028)$, and respondents who purchased a combination meal $(\mathrm{AOR}=1.23, \mathrm{p}=0.012)$.

\section{Discussion}

Numerous studies suggest that respondents purchase a similar number of calories pre- and post-calorie labeling [3-5]. This result has often been interpreted as suggesting that consumers do not use calorie-labeling information.

Researchers found that consumers in Philadelphia, which implemented calorie-labeling policies, were less likely to grossly underestimate calories (by $>500$ calories) post-labeling, relative to Baltimore, which did not implement such policies. These results suggest that at some level, consumers may incorporate labeling information, a novel result. Categorical accuracy for underestimation by $>100$ calories varied widely by subgroup, with improved accuracy among more educated consumers and those ordering small meals, and lower accuracy among women, consumers ordering large meals, and consumers ordering combination meals. No significant differences by race were found. Further research exploring why consumers choose to purchase a high number of calories despite increased awareness of the number of calories purchased is needed.

Perhaps most notably, respondents with a BA education or higher had a $75 \%$ reduction in odds for underestimating by $>100$ calories in Philadelphia post- versus pre-labeling (Table 3). This finding suggests that public health campaigns to promote understanding of calorie labeling may best be centered around less educated populations, who are less likely to report using posted information [2]. While females had 38\% increased odds for underestimating by $>100$ calories post-calorie labeling (Table 3 ), this finding may be tempered by an 8.1 percentage point increase in the proportion of females in Philadelphia post-calorie labeling ( $\mathrm{p}=0.010$, Table 1), compared with an insignificant change in the proportion of females in Baltimore $(p=0.053$, Table 1). We therefore would be cautious not to overinterpret differences in use of calorie labeling by gender, although some prior work in psychology has found greater calorie underestimation by women [25]. Additionally, while consumers could have purchased differently as a result of the survey or incentive (\$2), the data collection procedures were consistent across all periods and locations, suggesting that this should not influence the impact estimates [2].

We also found that the odds of underestimating calories post-calorie labeling declined in respondents who purchased $\leq$ median number of calories $(\mathrm{AOR}=0.54, \mathrm{p}<0.001)$ but increased in respondents who purchased $>$ median calories $(\mathrm{AOR}=1.27, \mathrm{p}=0.028)$ (Table 3$)$. Since respondents who purchased combination meals bought twice as many calories as other respondents (medians $=1340$ and 670 calories, respectively), it is possible that calorie labels for combination meals were more confusing. These calorie labels typically gave wider ranges ("500-2000 calories") that required individuals wanting further information to lookup calories for each item in the combination meal. Future research should consider whether providing more detailed information on combination meal calorie labels might improve overall accuracy.

\section{Appendix}

The change in calories purchased in Philadelphia postcalorie labeling legislation was assumed to derive from two potential factors, calorie labeling legislation or secular trends. To measure secular trends, researchers surveyed calories purchased in Baltimore, a control city, during the same time periods as for Philadelphia. Researchers assumed that the change in calories purchased in Baltimore would represent the secular trend, and any remaining change in calories purchased would be due to calorie labeling legislation. The difference in calories purchased in 
Table 3 Error in estimate of number of calories purchased, Philadelphia vs. Baltimore

\begin{tabular}{llllll}
\multicolumn{2}{l}{ Philadelphia } & & & \multicolumn{3}{l}{ Baltimore } & & Difference-in-Difference & \\
& Pre- & Post- & Post- & $\begin{array}{l}\text { Odds ratio } \\
(95 \% \mathrm{Cl})\end{array}$ & P
\end{tabular}

Percent

Error in estimate of number of calories (kcal) purchased

Full sample, correct within $100 \mathrm{kcal}$

Overestimated by $>100 \mathrm{kcal}$
Correctly estimated within $100 \mathrm{kcal}$
Underestimated by $>100 \mathrm{kcal}$

Full sample, correct within $250 \mathrm{kcal}$

Overestimated by $>250 \mathrm{kcal}$
Correctly estimated within $250 \mathrm{kcal}$
Underestimated by $>250 \mathrm{kcal}$

Full sample, correct within $500 \mathrm{kcal}$

Overestimated by $>500 \mathrm{kcal}$

Correctly estimated within $500 \mathrm{kcal}$

Underestimated $>500 \mathrm{kcal}$

Purchased $>\mathbf{8 5 0}$ kcal (median)

Overestimated by $>100 \mathrm{kcal}$

Correctly estimated within $100 \mathrm{kcal}$

Underestimated $>100 \mathrm{kcal}$

Purchased $\leq \mathbf{8 5 0} \mathrm{kcal}$ (median)

Overestimated by $>100 \mathrm{kcal}$

Correctly estimated within $100 \mathrm{kcal}$

Underestimated $>100 \mathrm{kcal}$

\section{Male}

Overestimated by $>100 \mathrm{kcal}$

Correctly estimated within $100 \mathrm{kcal}$

Underestimated $>100 \mathrm{kcal}$

\section{Female}

Overestimated by >100 kcal

Correctly estimated within $100 \mathrm{kcal}$

Underestimated $>100 \mathrm{kcal}$

Black

Overestimated by >100 kcal

Correctly estimated within $100 \mathrm{kcal}$

Underestimated $>100 \mathrm{kcal}$

\section{White}

Overestimated by $>100 \mathrm{kcal}$

Correctly estimated within $100 \mathrm{kcal}$

Underestimated $>100 \mathrm{kcal}$

\section{High school or less}

Overestimated by $>100 \mathrm{kcal}$

Correctly estimated within $100 \mathrm{kcal}$

Underestimated $>100 \mathrm{kcal}$

$\begin{array}{llll}11.9 & 14.4 & 25.6 & 15.3 \\ 18.9 & 15.5 & 10.2 & 14.2 \\ 69.2 & 70.0 & 64.2 & 70.5\end{array}$

5.3

14.2

70.5

$-5.4$

$0.90(0.67-1.21)$

0.48

$\begin{array}{llll}9.6 & 10.3 & 21.6 & 11.7\end{array}$

$\begin{array}{llll}32.6 & 34.8 & 29.7 & 33.9\end{array}$

$\begin{array}{llll}57.9 & 54.9 & 48.7 & 54.5\end{array}$

$-8.7$

$0.82(0.65-1.04)$

0.095

$\begin{array}{llll}6.8 & 5.6 & 14.5 & 7.3\end{array}$

$53.6 \quad 58.8$

39.6

58.8

35.6

53.3

7.3

55.6

$32.2 \quad 37.1$

$-8.8$

$0.75(0.73-0.77)$

$<0.001^{* * *}$

$\begin{array}{llll}9.8 & 11.1 & 25.0 & 8.5\end{array}$

7.3

4.8

3.4

8.5

6.2

82.9

84.1

71.6

85.3

$-12.5$

$1.27(1.03-1.56)$

$0.028^{*}$

$\begin{array}{llll}14.3 & 17.4 & 26.3 & 21.7\end{array}$

$31.7 \quad 25.2$

17.7

21.7

21.7

54.0

57.5

55.9

56.6

2.7

$0.54(0.37-0.78)$

$0.001^{* *}$

12.0

16.2

20.7

14.0

17.5

16.6

67.3

9.9

69.5

14.4

71.6

$-5.3$

$0.81(0.60-1.08)$

0.15

$11.4 \quad 13.2$

21.9

17.5

20.5

13.6

8.8

14.3

68.2

73.3

69.3

68.2

6.2

15.0

12.0

15.3

25.4

8.6

13.5

65.9

16.1

68.5

66.0

71.6

$-2.9$

0.96 (0.60-1.52)

0.86

$\begin{array}{ll}13.9 & 14.7 \\ 14.8 & 10.5 \\ 71.3 & 74.7\end{array}$

26.4

17.4

13.8

59.8

16.3

66.3

$-3.1$

1.29 (0.85-1.96)

0.22

$\begin{array}{llll}8.6 & 12.1 & 26.5 & 11.9 \\ 21.4 & 14.8 & 9.0 & 13.0 \\ 70.0 & 73.1 & 64.5 & 75.1\end{array}$

11.9

13.0

75.1

$-7.6$

$0.82(0.60-1.13)$

0.22 
Table 3 Error in estimate of number of calories purchased, Philadelphia vs. Baltimore (Continued)

\section{Some college or AA}

Overestimated by $>100 \mathrm{kcal}$

Correctly estimated within $100 \mathrm{kcal}$

Underestimated >100 kcal

BA or above

Overestimated by $>100 \mathrm{kcal}$

Correctly estimated within $100 \mathrm{kcal}$

Underestimated >100 kcal

\section{Food only}

Overestimated by $>100 \mathrm{kcal}$

Correctly estimated within $100 \mathrm{kcal}$

Underestimated $>100 \mathrm{kcal}$

\section{Beverage only}

Overestimated by $>100 \mathrm{kcal}$

Correctly estimated within $100 \mathrm{kcal}$

Underestimated $>100 \mathrm{kcal}$

\section{Purchased 1 item}

Overestimated by $>100 \mathrm{kcal}$

Correctly estimated within $100 \mathrm{kcal}$

Underestimated $>100 \mathrm{kcal}$

\section{Purchased $>1$ item}

Overestimated by $>100 \mathrm{kcal}$

Correctly estimated within $100 \mathrm{kcal}$

Underestimated $>100 \mathrm{kcal}$

Purchased combination meal

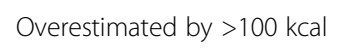

Correctly estimated within $100 \mathrm{kcal}$

Underestimated $>100 \mathrm{kcal}$

Did not purchase combination meal

Overestimated by $>100 \mathrm{kcal}$
Correctly estimated within $100 \mathrm{kcal}$
Underestimated $>100 \mathrm{kcal}$

Unadj.,Unadjusted. kcal: Calories.

${ }^{* * *} P<0.001$, ${ }^{* *} P<0.01$, ${ }^{*} P<0.05$.

Philadelphia, relative to the change in calories purchased in Baltimore, is sometimes called the "difference-in-difference." The regression model was as follows:

$$
\begin{aligned}
y= & a+\beta_{0} \times[\text { Philadelphia }]+\beta_{1} \times[\text { Post }]+\beta_{2} \\
& \times[\text { Philadelphia } * \text { Post }]+\delta \times[\mathbf{X}]+\varepsilon
\end{aligned}
$$

where $\alpha=$ constant; Philadelphia $=1$ if Philadelphia, 0 if Baltimore; Post $=1$ if post-calorie labeling legislation, 0 if pre-calorie labeling legislation; $\boldsymbol{X}=$ an array of all other independent variables (with a corresponding array of coefficient estimates $\delta$ ); and $\varepsilon=$ error term. $\beta_{2}$, the interaction between Philadelphia and postcalorie labeling legislation, represented the differencein-difference estimate.

\section{Competing interests}

The authors declare that they have no competing interests.

Authors' contributions

GBT was involved in conceptualizing the study, design, analysis plan, interpretation of results, and writing. BE was involved in conceptualizing the study, design,

interpretation and writing. Both authors read and approved the final manuscript.

\section{Acknowledgements}

This project was supported by grant number R01HL095935 from the NIH/ $\mathrm{NHLBI}$. The funders had no role in study design, data collection and analysis, decision to publish, or preparation of the manuscript. 
This study was completed while Dr. Taksler was with the Departments of Population Health and Medicine, New York University School of Medicine, New York, NY.

\section{Funding}

This project was supported by grant number R01HL095935 from the NIH/NHLBI.

\section{Author details}

${ }^{1}$ Medicine Institute, Cleveland Clinic, 9500 Euclid Avenue, G1-40F, Cleveland, $\mathrm{OH} 44195$, USA. ${ }^{2}$ Departments of Population Health and Medicine, New York University School of Medicine, New York, NY, USA. ${ }^{3}$ New York University Wagner School of Public Service, New York, NY, USA.

Received: 30 September 2013 Accepted: 2 July 2014

Published: 12 July 2014

\section{References}

1. 111th United States Congress: Patient Protection and Affordable Care Act. Washington, D.C.: United States Government Printing Office; 2010. H.R. 3590, PL 111-148, sec. 4205(b)(i)-(iii).

2. Elbel B, Mijanovich T, Dixon B, Abrams C, Weitzman B, Kersh R, Auchincloss $\mathrm{AH}$, Ogedegbe G: Calorie labeling, fast food purchasing and restaurant visits. Obesity 2013, 21(11):2172-2179.

3. Elbel B, Kersh R, Brescoll BL, Dixon LB: Calorie labeling and food choices: a first look at the effects on low-income people in New York City. Health Aff 2009, 28:w1110-w1121.

4. Elbel B, Gyamfi J, Kersh R: Child and adolescent fast-food choice and the influence of calorie labeling: a natural experiment. Int J Obes 2011, 35:493-500

5. Vadiveloo MK, Dixon LB, Elbel B: Consumer purchasing patterns in response to calorie labeling legislation in New York City. Int J Behav Nutr Phys Act 2011, 8:51.

6. Finkelstein EA, Strombotne KL, Chan NL, Krieger J: Mandatory menu labeling in one fast-food chain in King County, Washington. Am J Prev Med 2011, 40(2):122-127.

7. Tandon PS, Zhou C, Chan NL, Lozano P, Couch SC, Glanz K, Krieger J, Saelens BE: The impact of menu labeling on fast-food purchases for children and parents. Am J Prev Med 2011, 41(4):434-438.

8. Bollinger $B$, Leslie $P$, Sorensen $A$ : Calorie posting in chain restaurants. Am Econ J Econ Policy 2011, 3(1):91-128

9. Harnack L, French SA, Oakes JM, Story MT, Jeffery RW, Rydell SA: Effects of calorie labeling and value size pricing on fast food meal choices: results from an experimental trial. Int J Behav Nutr Phys Act 2008, 5:63.

10. Roberto C, Larsen P, Agnew H, Baik J, Brownell K: Evaluating the impact of menu labeling on food choices and intake. Am J Public Health 2010, 100:312-318.

11. Elbel B: Consumer estimation of recommended and actual calories at fast food restaurants. Obesity 2011, 19(10):1971-1978.

12. Wansink B, Painter JE, North J: Bottomless bowls: why visual cues of portion size may influence intake. Obesity 2005, 13(1):93-100.

13. Rolls BJ, Morris EL, Roe LS: Portion size of food affects energy intake in normal-weight and overweight men and women. Am J Clin Nutr 2002, 76(6):1207-1213.

14. Pettigrew $S$, Rosenberg M, Ferguson R: Consumers' (in)ability to estimate the energy content of unhealthy foods. Nutr Diet 2013, 70(4):307-311.

15. Ellison B, Lusk JL, Davis D: Looking at the label and beyond: the effects of calorie labels, health consciousness, and demographics on caloric intake in restaurants. Int J Behav Nutr Phys Act 2013, 10:21.

16. Brissette I, Lowenfels A, Noble C, Spicer D: Predictors of total calories purchased at fast-food restaurants: restaurant characteristics, calorie awareness, and use of calorie information. J Nutr Educ Behav 2013, 45(5):404-411.

17. Hawley KL, Roberto CA, Bragg MA, Liu PJ, Schwartz MB, Brownell KD: The science on front-of-package food labels. Public Health Nutr 2013, 16(3):430-439.

18. Roberto CA, Bragg MA, Schwartz MB, Seamans MJ, Musicus A, Novak N, Brownell KD: Facts up front versus traffic light food labels: a randomized controlled trial. Am J Prev Med 2012, 43(2):134-141.

19. Lichtenstein AH, Carson JS, Johnson RK, Kris-Etherton PM, Pappas A, Rupp L, Stitzel KF, Vafiadis DK, Fulgoni VL 3rd: Food-intake patterns assessed by using front-of-pack labeling program criteria associated with better diet quality and lower cardiometabolic risk. Am J Clin Nutr 2014, 99(3):454-462.
20. Cha E, Kim KH, Lerner HM, Dawkins CR, Bello MK, Umpierrez G, Dunbar SB: Health literacy, self-efficacy, food label use, and diet in young adults. Am J Health Behav 2014, 38(3):331-339.

21. Nagya RM: Determinants of consumers' use of nutritional information on food packages. J Agric Appl Econ 1996, 28(2):303-312.

22. Nagya RM, Lipinski D, Savur N: Consumers' use of nutritional labels while food shopping and at home. J Consum Aff 1998, 32(1):106-120.

23. Stran KA, Knol LL: Determinants of food label use differ by sex. J Acad Nutr Diet 2013, 113(5):673-679.

24. Johnson WG, Corrigan SA, Schlundt DG, Dubbert PM: Dietary restraint and eating behavior in the natural environment. Addict Behav 1990, 15(3):285-290

25. Mooney KM, DeTore J, Malloy KA: Perceptions of women related to food choice. Sex Roles 1994, 31(7-8):433-442.

\section{doi:10.1186/s12966-014-0091-2}

Cite this article as: Taksler and Elbel: Calorie labeling and consumer estimation of calories purchased. International Journal of Behavioral Nutrition and Physical Activity 2014 11:91.

\section{Submit your next manuscript to BioMed Central and take full advantage of:}

- Convenient online submission

- Thorough peer review

- No space constraints or color figure charges

- Immediate publication on acceptance

- Inclusion in PubMed, CAS, Scopus and Google Scholar

- Research which is freely available for redistribution

Submit your manuscript at www.biomedcentral.com/submit
C) Biomed Central 